\title{
Late-presenting congenital diaphragmatic hernia in an infant with tuberous sclerosis - case report and a review of the literature
}

\author{
Marta Komarowska, Ewa Matuszczak, Marcin Baran, Ewa Dzienis-Koronkiewicz, \\ Adam Hermanowicz, Wojciech Dębek
}

Department of Paediatric Surgery and Urology, Medical University of Bialystok, Poland

ABSTRACT

Tuberous sclerosis complex (TSC) is a rare, genetic syndrome, which is characterized by the occurrence of small, benign multilocalised hamartomas. The clinical manifestation of the disease is variable, from mild to life threating. This report presents a 9-month-old male baby suffering from TSC, which was diagnosed prenatally. The child was under constant medical, multidisciplinary monitoring. This boy presented skin lesions, hamartomas in the brain and heart, and observation toward hamartoma of the right retina. The infant was admitted to the hospital because of vomiting, fever and cough. Chest X-ray showed left diaphragmatic hernia with mediastinum shift. He underwent thoracoscopic hernia repair. The postoperative period was complicated by a left pneumothorax, atelectasis and pneumonia, but finally the child recovered and remains under ambulatory monitoring. Every pathological symptom must be imaged and diagnosed, despite good general condition.

KEY WORDS:

infant, congenital diaphragmatic hernia, tuberous sclerosis complex, radiologic symptoms.

\section{INTRODUCTION}

Tuberous sclerosis complex (TSC) is a genetic neurocutaneous syndrome that is characterized by multiple, mostly nonmalignant tumours called hamartomas, in many organs, such as the skin, eyes, brain, liver, kidney, heart and lungs [1]. TSC is a chronic, progressive, autosomal dominant disorder [2], and is caused by a mutation in TSC1 (hamartin) or TSC2 (tuberin) suppressor genes. The incidence rate of this syndrome is estimated at $1 / 6,000-1 / 10,000$ [3]. The clinical manifestation is variable. Most patients have skin lesions and seizures [4], however symptoms can be from mild to life threating. We want to present a rare case of a neonate suffering from
TSC and late-presenting congenital diaphragmatic hernia, which was successfully treated in our Department.

\section{CASE REPORT}

A 10-month-old male baby was admitted to the Emergency Room because of the cough lasting for a week, loss of appetite, and persistent vomiting for the last 2 days. The family physician diagnosed a viral infection the day before admission, but he did not perform additional tests.

The child suffered from TSC and thus was under constant multispecialty medical care. This syndrome was recognized prenatally, when MRI imaged small tumours in the brain and heart. Manifestation of TSC in this patient

\section{ADDRESS FOR CORRESPONDENCE:}

Ewa Matuszczak, Department of Paediatric Surgery and Urology, Medical University of Bialystok,

17 Waszyngtona St., 15-274 Białystok, Poland, ORCID: 0000-0003-2425-9589, e-mail: ewamat@tlen.pl 
was varied: first of all, neurological: cortical tubers, corti$\mathrm{cal} /$ subcortical tubers, subependymal nodules in the walls of ventricles and delayed myelination (images in MRI an CT); eyes: observation toward hamartoma of the right retina; heart: small multiple rhabdomyoma in both ventricles (in echosonography) caused asymptomatic multiple episodes of supraventricular extrasystoles (Holter monitoring). He was treated with vigabatrin because of infantile spasm. Family history was burdened with TSC. Four of his family members suffer from TSC: his mother, both siblings (10-year-old brother and 2-year-old brother) and maternal grandmother. There was no history of any trauma or accident.

His vital signs were: temperature $36.5^{\circ} \mathrm{C}$, respiratory rate 25 breaths/min, heart rate 100 beats/min., without cyanosis, oxygen saturation $\mathrm{SpO}_{2} 96 \%$. On examination, we found a weight deficiency ( $<3$ percentile), apathy and features of dehydration. There were also four small hypomelanoic macules in the lower limbs and buttocks. Chest auscultation revealed decreased breath sound on the left side. Our attention was also drawn to the scaphoid abdomen. Because pneumonia was suspected, X-ray was ordered. On the X-ray image, we saw bowel loops into the left side of the thorax with dislocation of the mediastinum to the right side and significantly reduced left lung (Fig. 1). Echosonography showed small multiple hyperechoic rhabdomyomas: three in the right ventricle (near the heart apex), with the largest diameters $12 \mathrm{~mm} \times 7 \mathrm{~mm}$, and two in the left ventricle (Fig. 2). These masses did not obstruct blood inflow or outflow. He was admitted to the Pediatric Surgery and Urology Department and after fluid resuscitation he was taken to the operating theatre. Before the operation, we started an intravenous antibiotic (co-amoxiclav). The patient underwent the thoracoscopic approach under general anaesthesia and ventilation of both lungs. A nasogastric tube was inserted, and the patient was placed on the right side with elevation of the left upper limb. The first trocar was placed under the scapula for $5 \mathrm{~mm}$ camera placement. Two more $3 \mathrm{~mm}$ trocars were inserted in the fifth intercostal space in the mid-clavicular and posterior axillary line. The insufflation of carbon dioxide was made with low pressures $6 \mathrm{~mm} \mathrm{Hg}$. There was a $4 \mathrm{~cm}$ postero-lateral defect in the left side of the diaphragmatic dome with the whole of the small and large intestines inside the chest. During operation we did not notice any evidence of a ruptured sac. According to Congenital Diaphragmatic Hernia Study Group (CDHSG) registry it was a defects are classified as "B". It involved $<50 \%$ of the chest wall [5]. The left lung was collapsed. Hernia content was gently reduced into the abdominal cavity. The diaphragm was closed with intra-corporally interrupted $2 / 0$ nonabsorbable sutures. The patient was ventilated and sedated for four days. Post-operative time was complicated by a left pneumothorax (treated by chest tube insertion) and with atelectasis and pneumonia of the left lung. He was treated in

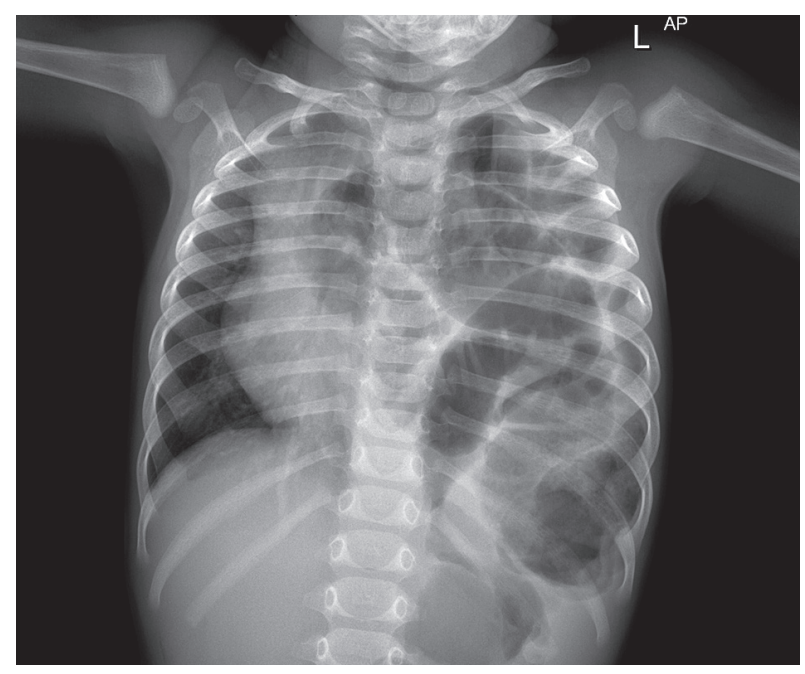

FIGURE 1. Left diaphragmatic hernia with mediastinum shift

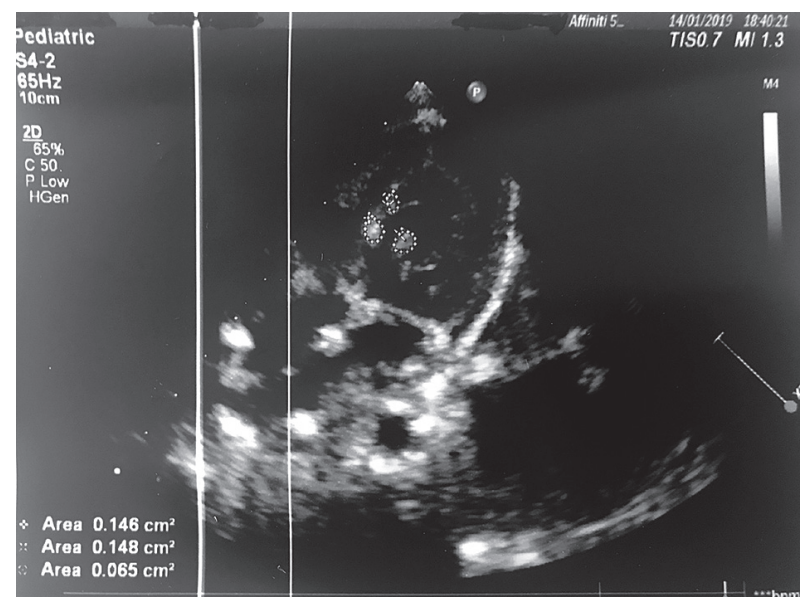

FIGURE 2. Hamartomas in the heart

the Pediatric Intensive Care Unit, but after that, the boy's recovery was complete. He was discharged on the $24^{\text {th }}$ day after surgical repair. He is still under follow-up care. His developmental parameters are normal.

\section{DISCUSSION}

The incidence of congenital diaphragmatic hernia $(\mathrm{CDH})$ is approximately $1 / 3,000$ live births [6]. The aetiology of $\mathrm{CDH}$ is still unclear. Genetic causes of $\mathrm{CDH}$ have been noticed in around $30 \%$ of patients [7]. Approximately $40 \%$ of $\mathrm{CDH}$ cases are associated with other anomalies [7] like brain, heart, renal and genitourinary disorders. In more than $50 \%$ of cases it could be diagnosed prenatally [8]. We can postulate that if our patient underwent prenatal MRI imaging it would seem plausible that the $\mathrm{CDH}$ defect would have been diagnosed prenatally. A newborn with $\mathrm{CDH}$ often presents severe respiratory distress. Whereas, in late-presenting $\mathrm{CDH}$, symptoms could be either acute or chronic. Gastrointestinal symptoms occur most often, but none of the complains are specific for late-presenting $\mathrm{CDH}$. In one study, gastrointestinal prob- 
lems were seen more commonly in $\mathrm{CDH}$ on the left side [9]. In the case of a late diagnosis, hernia is more common on the right side [10], and this is explained by the fact that the liver is a natural, mechanical barrier to dislocation of the intestines to the chest cavity. Late-presenting congenital diaphragmatic hernia accounts for $10-13 \%$ all children with $\mathrm{CDH}$ [10]. According to the literature, it is more common in males than females [11] and usually it is left side postero-lateral hernia. In most cases, it is an isolated congenital defect. In our case, chest X-ray was enough to make the right diagnosis, but if the image is ambiguous, chest and abdominal CT can help make the right diagnosis.

In the literature, there was only one case of TSC in a neonate presented as diaphragmatic hernia [12] and we can also find mention about two other cases. One it was a 7.5-year-old girl [13], who presented with a diaphragmatic hernia, multiple cardiac rhabdomyomas and a horseshoe kidney with mutation in the TSC2 gene. The second case of TSC connected with CDH was a preterm neonate, in whom diaphragmatic hernia was found during autopsy, in addition to cardiac rhabdomyomas and intestine malrotation. Ohri et al. who described case of tuberous sclerosis in a newborn who had also multiple rhabdomyomas of heart and diaphragmatic hernia, associate the presence of this congenital malformations with multi-germ layer dysplasia [12]. Defects in morphogenesis associated with $\mathrm{CDH}$ are heterogeneous, and seemingly related to numerous pathogenetic mechanisms. Most often abnormalities involve heart, brain, genitourinary system, craniofacial region, or limbs [5].

Most patients with the tuberous sclerosis complex suffer from seizures and differential skin lesions [14]. Signs of TSC in the early infant period are usually mild, and the most common initial disorders in small children are cardiac rhabdomyomas and hypomelanotic macules [15]. As in our patient, if the diagnosis of TSC is made prenatally, careful monitoring must be implemented. According to new diagnostic criteria from 2012 [16] pulmonary manifestation, in the form of lymphangioleiomyomatosis (LAM), is one of the major criteria of TSC. LAM is characterized by cysts and excessive proliferation of the smooth muscles, which replace normal pulmonic alveoli, and it is more commonly seen in women [17]. Patients with LAM are at higher risk of spontaneous pneumothorax [18]. Second pulmonary disease is multifocal micronodular pneumocyte hyperplasia (MMPH) [19], which is a rare, benign hamartomatous subtype of type II pneumocyte proliferation [19]. In contrast to LAM, MMPH occurs both in women and in men. Postoperative complications in our patient could be connected with a baseline disorder and strict future observation should be focused on the lungs. On the other hand, it cannot be ruled out that the postoperative pneumothorax was from residual carbo dioxide from thoracoscopy approach. There is still debate about benefits and risks of chest tube placement.

\section{CONCLUSIONS}

Clinical manifestation of TSC is heterogeneous and can create diagnostic problems. Patients with tuberous sclerosis complex should be under strict medical monitoring. What is important, every pathological symptom must be imaged and diagnosed, despite good general condition. Diagnosis of congenital diaphragmatic hernia after the neonate period is rare, but possible. Symptoms are non-specific and mainly relate to gastrointestinal or respiratory systems. The surgical correction of $\mathrm{CDH}$ may be performed using open or minimal invasive surgery. The approach could be transthoracic or through the abdomen. In late-presenting $\mathrm{CDH}$ patient are usually without pulmonary hypoplasia cardio-pulmonary instability, therefore these patients seem to be good candidates for minimal invasive surgery.

\section{DISCLOSURE}

The authors declare no conflict of interest.

\section{REFERENCES}

1. Portocarrero LKL, Quental KN, Samorano LP, et al. Tuberous sclerosis complex: review based on new diagnostic criteria. An Bras Dermatol 2018; 93: 323-331.

2. Crino PB, Nathanson KL, Henske EP. The tuberous sclerosis complex. N Engl J Med 2006; 355: 1345-1356.

3. O'Callaghan FJ, Shiell AW, Osborne JP, Martyn CN. Prevalence of tuberous sclerosis estimated by capture-recapture analysis. Lancet 1998; 351: 1490.

4. Wheless JW, Almoazen H. A novel topical rapamycin cream for the treatment of facial angiofibromas in tuberous sclerosis complex. J Child Neurol 2013; 28: 933-936.

5. Harting MT, Lally KP. The Congenital Diaphragmatic Hernia Study Group registry update. Semin Fetal Neonatal Med 2014; 19: 370375.

6. Doyle NM, Lally KP. The CDH Study Group and advances in the clinical care of the patient with congenital diaphragmatic hernia. Semin Perinatol 2004; 28: 174-184.

7. Yu L, Sawle AD, Wynn J, et al. Increased burden of de novo predicted deleterious variants in complex congenital diaphragmatic hernia. Hum Mol Genet 2015; 24: 4764-4773.

8. Gallot D, Boda C, Ughetto S, et al. Prenatal detection and outcome of congenital diaphragmatic hernia: a French registry-based study. Ultrasound Obstet Gynecol 2007; 29: 276-283.

9. Kitano Y, Lally KP, Lally PA, Group CDHS. Late-presenting congenital diaphragmatic hernia. J Pediatr Surg 2005; 40: 1839-1843.

10. Al Ghafri M, Al Sidairi I, Nayar M. Late presentation of congenital diaphragmatic hernia: a case report. Oman Med J 2014; 29 : 223-225.

11. Bagłaj M. Late-presenting congenital diaphragmatic hernia in children: a clinical spectrum. Pediatr Surg Int 2004; 20: 658-669. 
12. Ohri GL, DeVenecia R, Acs H. Tuberous sclerosis presenting as diaphragmatic hernia in a newborn. Dev Med Child Neurol 1980; 22: 509-512.

13. Niemi AK, Northrup H, Hudgins L, Bernstein JA. Horseshoe kidney and a rare TSC2 variant in two unrelated individuals with tuberous sclerosis complex. Am J Med Genet A 2011; 155A: 25342537.

14. Tu J, Foster RS, Bint LJ, Halbert AR. Topical rapamycin for angiofibromas in paediatric patients with tuberous sclerosis: follow up of a pilot study and promising future directions. Australas J Dermatol 2014; 55: 63-69.

15. Davis PE, Filip-Dhima R, Sideridis G, et al. Presentation and Diagnosis of Tuberous Sclerosis Complex in Infants. Pediatrics 2017; 140: e20164040.

16. Krueger DA, Northrup H, Group ITSCC. Tuberous sclerosis complex surveillance and management: recommendations of the 2012 International Tuberous Sclerosis Complex Consensus Conference. Pediatr Neurol 2013; 49: 255-265.

17. Rosset C, Netto CBO, Ashton-Prolla P. TSC1 and TSC2 gene mutations and their implications for treatment in Tuberous Sclerosis Complex: a review. Genet Mol Biol 2017; 40: 69-79.

18. Cooley J, Lee YCG, Gupta N. Spontaneous pneumothorax in diffuse cystic lung diseases. Curr Opin Pulm Med 2017; 23: 323-333.

19. Gupta N, Henske EP. Pulmonary manifestations in tuberous sclerosis complex. Am J Med Genet C Semin Med Genet 2018; 178: 326-337. 